\title{
Improved Characteristics of InGaZnO Thin-Film Transistor by using Fluorine Implant
}

L. X. Qian, ${ }^{a}$ W. M. Tang, ${ }^{b}$ and P. T. Lai ${ }^{a, z}$

${ }^{a}$ Department of Electrical and Electronic Engineering, The University of Hong Kong, Pokfulam Road, Hong Kong, Hong Kong

${ }^{\mathrm{b}}$ Department of Applied Physics, The Hong Kong Polytechnic University, Hung Hom, Kowloon, Hong Kong

Index Terms

Amorphous InGaZnO (a-IGZO), thin-film transistor (TFT), fluorine, implant

${ }^{\mathrm{z} E-m a i l: ~ 1 a i p @ e e e . h k u . h k}$. 


\section{ABSTRACT}

The effects of fluorine incorporation in amorphous $\mathrm{InGaZnO}$ by ion implant on the characteristics of InGaZnO thin-film transistor have been investigated. The electrical characteristics of thin-film transistor can be improved by this treatment due to increase of carrier concentration and passivation of defects in InGaZnO. Consequently, the saturation carrier mobility can be increased from $25.2 \mathrm{~cm} / \mathrm{V} \cdot \mathrm{s}$ to a high value of $34.0 \mathrm{~cm}^{2} / \mathrm{V} \cdot \mathrm{s}$, and the output current can be nearly doubled. However, device degradation is observed for very high fluorine dose above $1.0 \times 10^{15} / \mathrm{cm}^{2}$, possibly ascribed to excessive interstitial fluorine atoms and too many fluorine-induced electrons in InGaZnO. 
Over the years, amorphous InGaZnO (a-IGZO) thin-film transistors (TFTs) have been extensively investigated for applications in various flat-panel displays (FPDs), including electronic papers, organic light-emitting-diode displays (OLEDs) and liquid crystal displays (LCDs). ${ }^{1,2}$ Compared to conventional amorphous-silicon or organic TFTs with a field-effect carrier mobility of $\sim 1 \mathrm{~cm}^{2} / \mathrm{V} \cdot \mathrm{s},{ }^{3,4}$ a-IGZO TFTs typically exhibit a mobility higher than $10 \mathrm{~cm}^{2} / \mathrm{V} \cdot \mathrm{s} .{ }^{5,6}$ In addition, a-IGZO TFTs offer better uniformity in device characteristics than polycrystalline silicon TFTs, and have much higher transparency to visible light than all the silicon-based devices. ${ }^{7}$

In order to meet the requirement of developing advanced display technologies, for example, largepanel LCDs, OLEDs and high-frame-rate three-dimensional (3D) displays, higher carrier motility is required for a-IGZO TFTs. It is well-known that carrier transport in a-IGZO is by percolation conduction, and a potential barrier distribution around the conduction-band bottom is formed due to its disordered structure. If the electron concentration in a-IGZO is increased, the potential barrier will be reduced, resulting in an increase in the carrier mobility of a-IGZO TFTs. ${ }^{2,8}$ Accordingly, doping with other element has been adopted to increase the electron concentration in a-IGZO and thus the carrier mobility of a-IGZO TFTs. For example, high-pressure hydrogen annealing was reported to realize improved electrical characteristics of a-IGZO TFTs, including increased saturation carrier mobility $\left(\mu_{\text {sat }}\right)$ from $0.7 \mathrm{~cm}^{2} / \mathrm{V} \cdot \mathrm{s}$ to $7.4 \mathrm{~cm}^{2} / \mathrm{V} \cdot \mathrm{s}$ and reduced threshold voltage $\left(\mathrm{V}_{\mathrm{TH}}\right)$ from $5.6 \mathrm{~V}$ to $2.2 \mathrm{~V}$, due to the increase of electron concentration in a-IGZO after hydrogen incorporation. ${ }^{8}$ On the other hand, Seo et al. adopted fluorine doping by spin coating of metal-fluoride $\left(\mathrm{ZnF}_{2}\right.$ and $\left.\mathrm{InF}_{3}\right)$ precursor aqueous solution to improve the carrier mobility and stability of InZnO TFTs. ${ }^{9}$ However, the reported InZnO TFTs had a low $\mu_{\mathrm{sat}}$ of $4.1 \mathrm{~cm}^{2} / \mathrm{V} \cdot \mathrm{s}$ and a high $\mathrm{V}_{\mathrm{TH}}$ of $5.5 \mathrm{~V}$ even after fluorine incorporation, possibly due to the solution process.

In this work, fluorine doping is introduced to a-IGZO TFTs through ion implant, and accordingly the effects of fluorination on the characteristics of a-IGZO TFTs are investigated both qualitatively and 
quantitatively. Furthermore, high-k material has been widely applied as gate dielectric in a-IGZO TFTs in order to reduce their operating voltage. ${ }^{10,11}$ Among them, HfLaO is one of the most promising dielectric materials due to its superior properties, including high dielectric constant, good thermal stability and low trap density, ${ }^{12}$ and thus is selected as the gate dielectric for the a-IGZO TFTs in this work.

The schematic diagrams of the device cross section during fluorine ion implant and after all the fabrication steps are shown in Figure 1 (a) and Figure 1 (b), respectively. In this structure, p-type (100) silicon with a resistivity of $0.01 \sim 0.02 \Omega \cdot \mathrm{cm}$ acts as both the substrate and gate electrode of each sample. Firstly, the conventional RCA (Radio Corporation of America) method was used to remove the organic and ionic contaminants on the substrate. ${ }^{13,14}$ Secondly, deposition of a 40-nm HfLaO film was done by means of a sputtering system with a radio-frequency (RF) power of $40 \mathrm{~W}$ and a metal target of HfLa (with 40\% lanthanum). Moreover, the sputtering process was conducted in a mixed ambient of Ar plus $\mathrm{O}_{2}\left(\mathrm{Ar}: \mathrm{O}_{2}=24 \mathrm{sccm}: 6 \mathrm{sccm}\right)$. Thirdly, an annealing treatment at $400{ }^{\circ} \mathrm{C}$ in an ambient of $\mathrm{N}_{2}$ for $10 \mathrm{~min}$ with a gas flow rate of $500 \mathrm{ml} / \mathrm{min}$ followed in order to improve the quality of the HfLaO film. Subsequently, each sample received a deposition of a 60-nm a-IGZO active layer through RF sputtering from a ceramic target $\left(\mathrm{Ga}_{2} \mathrm{O}_{3}: \mathrm{In}_{2} \mathrm{O}_{3}: \mathrm{ZnO}=1: 1: 1\right)$ in an $\mathrm{Ar} / \mathrm{O}_{2}$ mixed ambient. Then, some samples were treated by fluorine ion implant at a fixed energy of $20 \mathrm{keV}$ and an angle of 7 degree, with a dose of $3.0 \times 10^{14} / \mathrm{cm}^{2}, 1.0 \times 10^{15} / \mathrm{cm}^{2}, 3.0 \times 10^{15} / \mathrm{cm}^{2}$ and $1.0 \times 10^{16} / \mathrm{cm}^{2}$, respectively. In addition, a control sample without fluorine incorporation was also fabricated for comparison purpose. After that, a lift-off process was utilized to form the source/drain electrodes, which were composed of 20-nm Ti and 80-nm $\mathrm{Au}$ deposited by means of electron-beam evaporation. Finally, all the samples were annealed in a forming-gas $\left(\mathrm{N}_{2}: \mathrm{H}_{2}=95: 5\right)$ ambient at $350{ }^{\circ} \mathrm{C}$ for $20 \mathrm{~min}$ so that the contact resistance of the source/drain electrodes was reduced. In addition, metal-insulator-semiconductor (MIS) capacitor was also prepared beside the transistor in order to monitor the gate-oxide capacitance per unit area $\left(\mathrm{C}_{\mathrm{ox}}\right.$, 
equal to $0.252 \mu \mathrm{F} / \mathrm{cm}^{2}$ in this work). For each TFT device, the channel width (W) and channel length (L) were $100 \mu \mathrm{m}$ and $30 \mu \mathrm{m}$ respectively, and all the processing steps except the annealings were conducted at room temperature.

The current-voltage (I-V) curve of the TFTs and the 1-MHz capacitance-voltage (C-V) characteristics of the capacitors were measured by a HP 4145B semiconductor parameter analyzer and a HP 4284A precision LCR meter, respectively. Besides, the physical thicknesses of HfLaO and a-IGZO were measured by a multi-wavelength ellipsometer. All the measurements were conducted in a lighttight, electrically-shielded and room-temperature environment.

Figure 2 exhibits the secondary ion-mass spectrometry (SIMS) depth profile for the aIGZO/HfLaO/Si stacks implanted with different fluorine doses. The ordinate refers to the atom intensity while the abscissa is about the acquisition time, which reflects the depth from the surface of a-IGZO. As shown in Figure 2, fluorine has been doped in a-IGZO through ion implant, and the fluorine distribution is similar for all the fluorinated samples due to the same implant energy. For each dose, the concentration of fluorine atom is relatively uniform from the surface of a-IGZO to its middle, and then starts to decrease towards the a-IGZO/HfLaO interface.

Figure 3 shows the atomic force microscopy (AFM) results of the a-IGZO/HfLaO/Si stacks implanted with different fluorine doses for a measurement area of $1 \mu \mathrm{m} \times 1 \mu \mathrm{m}$. As shown in Figure 3 (a), the control sample exhibits the roughest a-IGZO surface, with a RMS value of $2.34 \mathrm{~nm}$. With the treatment of fluorine ion implant, the surface roughness of a-IGZO is significantly reduced, which is more obvious for higher dose. Consequently, the RMS value of the fluorinated samples is $1.28 \mathrm{~nm}, 1.16$ $\mathrm{nm}, 0.58 \mathrm{~nm}$ and $0.31 \mathrm{~nm}$ for a dose of $3.0 \times 10^{14} / \mathrm{cm}^{2}, 1.0 \times 10^{15} / \mathrm{cm}^{2}, 3.0 \times 10^{15} / \mathrm{cm}^{2}$ and $1.0 \times 10^{16} / \mathrm{cm}^{2}$, respectively. The correlation between the RMS value and the fluorine dose is exhibited in Figure 3 (f), which shows a strong consistency of the improvement in the surface roughness of a-IGZO induced by fluorine incorporation. This finding proves that fluorine atoms can effectively passivate the defects in a- 
IGZO, e.g. by combining with its dangling bonds. ${ }^{15}$ Moreover, it is believed that a smoother a-IGZO surface could reveal improved quality of the channel/dielectric interface, ${ }^{16}$ which is important for better electrical characteristics of a-IGZO TFTs.

Figure 4 (a) shows the transfer characteristics of the a-IGZO TFTs implanted with different fluorine doses: drain current $\left(\mathrm{I}_{\mathrm{D}}\right)$ vs. gate-to-source voltage $\left(\mathrm{V}_{\mathrm{GS}}\right)$, and $\mathrm{I}_{\mathrm{D}}{ }^{1 / 2}$ vs. $\mathrm{V}_{\mathrm{GS}}$ at a drain-to-source voltage $\left(\mathrm{V}_{\mathrm{DS}}\right)$ of $5 \mathrm{~V}$. The electrical parameters of the devices, including $\mu_{\mathrm{sat}}, \mathrm{V}_{\mathrm{TH}}$, subthreshold swing (SS) and on-off current ratio $\left(\mathrm{I}_{\mathrm{on}} / \mathrm{I}_{\text {off }}\right)$ are extracted from Figure 4 (a) and listed in Table I . Among them, $\mu_{\text {sat }}$ and $\mathrm{V}_{\mathrm{TH}}$ are calculated from a linear fitting to the plot of $\mathrm{I}_{\mathrm{D}}{ }^{1 / 2}$ versus $\mathrm{V}_{\mathrm{GS}}$, which is based on the I-V equation of field-effect transistor operating in the saturation region.

$$
\mathrm{I}_{\mathrm{D}}=\left(\mu_{\mathrm{sat}} \mathrm{C}_{\mathrm{ox}} \mathrm{W} / 2 \mathrm{~L}\right)\left(\mathrm{V}_{\mathrm{GS}}-\mathrm{V}_{\mathrm{TH}}\right)^{2}
$$

As shown in Figure 4 (b), compared to the control sample $\left(\mu_{\text {sat }}=25.2 \mathrm{~cm}^{2} / \mathrm{V} \cdot \mathrm{s}\right), \mu_{\text {sat }}$ of the fluorinated sample increases almost linearly with the dose from $0 / \mathrm{cm}^{2}$ to $1.0 \times 10^{15} / \mathrm{cm}^{2}$, and then starts to decrease. Accordingly, the highest value of $\mu_{\mathrm{sat}}\left(34.0 \mathrm{~cm}^{2} / \mathrm{V} \cdot \mathrm{s}\right)$ is achieved by the sample with a fluorine dose of $1.0 \times 10^{15} / \mathrm{cm}^{2}$. It is well known that fluorine and oxygen have similar ionic radius, and the zinc-fluorine chemical bonding $(\mathrm{Zn}-\mathrm{F})$ is stronger than the zinc-oxygen chemical bonding $(\mathrm{Zn}-\mathrm{O})$. Therefore, it is easy to cause the substitution of the oxygen atoms in the lattice $\left(\mathrm{O}_{\mathrm{o}}^{\mathrm{x}}\right)$ of a-IGZO by the fluorine atoms derived from the implant. Moreover, the difference in electrovalency between oxygen ion $\left(\mathrm{O}^{2-}\right)$ and fluorine ion $\left(\mathrm{F}^{-}\right)$means that the substitution of an oxygen ion by a fluorine ion can generate a free electron: ${ }^{9}$

$$
\mathrm{O}_{\mathrm{O}}^{\mathrm{X}}+\mathrm{F}^{-} \rightarrow \mathrm{F}_{\mathrm{O}}^{\bullet}+\mathrm{e}^{-}
$$

Therefore, the electron concentration in a-IGZO is increased, resulting in enhanced carrier mobility for a-IGZO TFTs. In addition, the increase of electron concentration in a-IGZO is further revealed by the $\mathrm{V}_{\mathrm{TH}}$ reduction of the fluorinated samples compared to the control sample in Table I. On the other hand, 
the passivation effect of fluorine on the defects in a-IGZO (including both the a-IGZO bulk and the aIGZO/HfLaO interface), e.g. the dangling bonds normally acting as electron trap sites in a-IGZO TFTs, is another important factor for increasing the carrier mobility due to the suppression of trap-induced scattering on channel electrons. This passivation effect has been supported by the AFM results which exhibit a continual improvement in the surface roughness of a-IGZO with increasing fluorine dose. Furthermore, it is believed that the SS of a-IGZO TFTs can be affected by the trap density at/near the aIGZO/dielectric interface. ${ }^{1,17}$ Hence, the reduction of SS after fluorine incorporation, as reflected by the comparison between the fluorinated sample with a dose of $3.0 \times 10^{14} / \mathrm{cm}^{2}(\mathrm{SS}=0.29 \mathrm{~V} / \mathrm{dec})$ or $1.0 \times 10^{15}$ $/ \mathrm{cm}^{2}(\mathrm{SS}=0.28 \mathrm{~V} / \mathrm{dec})$ and the control sample $(\mathrm{SS}=0.32 \mathrm{~V} / \mathrm{dec})$, reveals the decrease in the trap density at/near the a-IGZO/HfLaO interface, further supporting the passivation effect of fluorine on the defects in a-IGZO. Due to the improvements in $\mu_{\text {sat }}$ and $V_{\mathrm{TH}}$, a continual increase in the $\mathrm{I}_{\mathrm{on}} / \mathrm{I}_{\mathrm{off}}$ of the aIGZO TFT is also achieved as the fluorine dose increases from $0 / \mathrm{cm}^{2}$ to $1.0 \times 10^{15} / \mathrm{cm}^{2}$. However, the device performance, including $\mu_{\text {sat }}$, SS and $\mathrm{I}_{\mathrm{on}} / \mathrm{I}_{\mathrm{off}}$, starts to degrade for fluorine dose over $1.0 \times 10^{15} / \mathrm{cm}^{2}$, possibly because excessive fluorine atoms could act as interstitial defects rather than passivators. ${ }^{18}$ In addition, for very high fluorine dose, the electron and defect concentrations in the IGZO film can become high enough to induce significant current flow in its bulk (loss of channel-current modulation by the $\mathrm{V}_{\mathrm{GS}}$ sweeping), ${ }^{19}$ as demonstrated by the hump in the subthreshold region of the transfer curve shown in Figure 4 (a), and accordingly the SS of TFT increases.

Figure 5 displays the output characteristics of the a-IGZO TFTs implanted with different fluorine doses. All the samples clearly exhibit n-type enhancement mode. Moreover, $I_{D}$ increases linearly with $\mathrm{V}_{\mathrm{DS}}$ in the region of low $\mathrm{V}_{\mathrm{DS}}$, and current saturation can be observed in the region of high $\mathrm{V}_{\mathrm{DS}}$. Due to the improvements in $\mu_{\text {sat }}$ and $\mathrm{V}_{\mathrm{TH}}$, the output current of the fluorinated sample increases significantly with the dose from $0 / \mathrm{cm}^{2}$ to $1.0 \times 10^{15} / \mathrm{cm}^{2}$. As shown in Figure $5(\mathrm{f})$, under the condition of $\mathrm{V}_{\mathrm{DS}}=8 \mathrm{~V}$ and $\mathrm{V}_{\mathrm{GS}}=10 \mathrm{~V}$, the output current of the a-IGZO TFT with a fluorine dose of $1.0 \times 10^{15} / \mathrm{cm}^{2}(660 \mu \mathrm{A})$ is 
nearly twice that of the control sample $(366 \mu \mathrm{A})$. On the other hand, performance degradation of the aIGZO TFT can also be observed from the output characteristics when the fluorine dose is higher than $1.0 \times 10^{15} / \mathrm{cm}^{2}$. In addition, since the phenomenon of current crowding is present in Figure 5 , the forming-gas annealing needs to be prolonged or done at higher temperature so as to improve the electrical contact between the source/drain electrode and a-IGZO.

In this work, TFT is fabricated on a-IGZO film implanted with fluorine, and accordingly the effects of fluorine incorporation in a-IGZO on the characteristics of a-IGZO TFT have been investigated both qualitatively and quantitatively. It is found that the electrical characteristics of the a-IGZO TFT, including $\mu_{\mathrm{sat}}, \mathrm{V}_{\mathrm{TH}}, \mathrm{I}_{\mathrm{on}} / \mathrm{I}_{\mathrm{off}}$, SS and output current, can be effectively improved by the fluorine implant due to the dual effects of fluorine incorporation in a-IGZO (increased carrier concentration and defect passivation). Accordingly, increased $\mu_{\mathrm{sat}}($ from $25.2 \mathrm{~cm} / \mathrm{V} \cdot \mathrm{s}$ to a high value of $34.0 \mathrm{~cm} / \mathrm{V} \cdot \mathrm{s}$ ) and almost doubled output current are achieved. However, the device performance starts to degrade when the fluorine dose is over $1.0 \times 10^{15} / \mathrm{cm}^{2}$, probably due to the formation of fluorine interstitials or excessively high electron concentration in the a-IGZO film heavily doped with fluorine. In summary, a-IGZO fluorination by ion implant is an effective and reproducible method to make high-performance TFT for applications in various advanced FPDs.

\section{Acknowledgment}

This work is supported by the University Development Fund (Nanotechnology Research Institute, 00600009) of the University of Hong Kong. 


\section{References}

1. J. S. Park, W. J. Maeng, H. S. Kim, and J.S. Park, Thin Sol. Films, 520, 1679 (2012).

2. T. Kamiya, K. Nomura, and H. Hosono, Sci. Technol. Adv. Mater., 11, 044305 (2010).

3. R. A. Street, Adv. Mater., 21, 2007 (2009).

4. L. F. Deng, P. T. Lai, W. B. Chen, J. P. Xu, Y. R. Liu, H. W. Choi, and C. M. Che, IEEE Electron Dev. Lett., 32, 93 (2011).

5. H. Yabuta, M. Sano, K. Abe, T. Aiba, T. Den, H. Kumomi, K. Nomura, T. Kamiya, and H. Hosono, Appl. Phys. Lett., 89, 112123 (2006).

6. L. X. Qian, P. T. Lai, and W. M. Tang, Appl. Phys. Lett., 104, 123505 (2014).

7. K. Nomura, H. Ohta, A. Takagi, T. Kamiya, M. Hirano, and H. Hosono, Nature, 432, 488 (2004).

8. S. I. Oh, G. Choi, H. Hwang, W. Lu, and J. H. Jang, IEEE Trans. Electr. Dev., 60, 2537 (2013).

9. J. S. Seo, J. H. Jeon, Y. H. Hwang, H. Park, M. Ryu, S. H. K. Park, and B. S. Bae, Sci. Rep., 3, 1 (2013).

10. J. S. Lee, S. Chang, S. M. Koo, and S. Y. Lee, IEEE Electron Dev. Lett., 31, 225 (2010).

11. J. B. Kim, C. F. Hernandez, and B. Kippelen, Appl. Phys. Lett., 93, 242111 (2008).

12. N. C. Su, S. J. Wang, C. C. Huang, Y. H. Chen, H. Y. Huang, C. K. Chiang, and A. Chin, IEEE Electron Dev. Lett., 31, $680(2010)$.

13. W. Kern and D. A. Poutinen, RCA Rev., 31, 187 (1970).

14. W. Kern, J. Electrochem.Soc., 137, 1887 (1990).

15. H. Y. Xu, Y. C. Liu, R. Mu, C. L. Shao, Y. M. Lu, D. Z. Shen, and X. W. Fan, Appl. Phys. Lett., 86, 123107 (2005).

16. L. Zhang, J. Li, X. W. Zhang, X. Y. Jiang, and Z. L. Zhang, Appl. Phys. Lett., 95, 072112 (2009).

17. J. K. Jeong, J. H. Jeong, H. W. Yang, J. S. Park, Y. G. Mo, and H. D. Kim, Appl. Phys. Lett., 91, 113505 (2007).

18. B. Liu, M. Gu, X. Liu, S. Huang, and C. Ni, Appl. Phys. Lett., 97, 122101 (2010).

19. N. Huby, S. Ferrari, E. Guziewicz, M. Godlewski, and V. Osinniy, Appl. Phys. Lett., 92, 023502 (2008). 
TABLE I. Extracted electrical parameters of the a-IGZO TFT's.

\begin{tabular}{cccccc}
\hline \hline $\mathrm{F}$ dose $\left(/ \mathrm{cm}^{2}\right)$ & 0 & $3.0 \times 10^{14}$ & $1.0 \times 10^{15}$ & $3.0 \times 10^{15}$ & $1.0 \times 10^{16}$ \\
\hline$\mu_{\mathrm{sat}}\left(\mathrm{cm}^{2} / \mathrm{V} \cdot \mathrm{s}\right)$ & 25.2 & 27.5 & 34.0 & 28.4 & 20.3 \\
$\mathrm{~V}_{\mathrm{TH}}(\mathrm{V})$ & 4.8 & 4.0 & 4.1 & 4.0 & 2.7 \\
$\mathrm{SS}(\mathrm{V} / \mathrm{dec})$ & 0.32 & 0.29 & 0.28 & 0.85 & 1.22 \\
$\mathrm{I}_{\mathrm{on}} / \mathrm{I}_{\text {off }}$ & $1.8 \times 10^{5}$ & $2.5 \times 10^{5}$ & $3.7 \times 10^{5}$ & $3.3 \times 10^{5}$ & $1.9 \times 10^{5}$ \\
Output current $(\mu \mathrm{A})$ & 366 & 455 & 660 & 504 & 440 \\
\hline \hline
\end{tabular}




\section{Figure captions}

Figure 1. Schematic diagrams of the device cross section: (a) during fluorine ion implant; (b) a-IGZO TFT with $\mathrm{HfLaO}$ gate dielectric.

Figure 2. SIMS depth profile for a-IGZO/HfLaO/Si stacks implanted with different fluorine doses.

Figure 3. AFM results of a-IGZO/HfLaO/Si stacks implanted with different fluorine doses: (a) $0 / \mathrm{cm}^{2}$; (b) $3.0 \times 10^{14} / \mathrm{cm}^{2}$; (c) $1.0 \times 10^{15} / \mathrm{cm}^{2}$; (d) $3.0 \times 10^{15} / \mathrm{cm}^{2}$; (e) $1.0 \times 10^{16} / \mathrm{cm}^{2}$; (f) RMS value versus fluorine dose.

Figure 4. (a) Transfer characteristics of a-IGZO TFTs implanted with different fluorine doses; (b) $\mu_{\text {sat }}$ versus fluorine dose.

Figure 5. Output characteristics of a-IGZO TFTs implanted with different fluorine doses: (a) $0 / \mathrm{cm}^{2}$; (b) $3.0 \times 10^{14}$ $/ \mathrm{cm}^{2}$; (c) $1.0 \times 10^{15} / \mathrm{cm}^{2}$; (d) $3.0 \times 10^{15} / \mathrm{cm}^{2}$; (e) $1.0 \times 10^{16} / \mathrm{cm}^{2}$; (f) $\mathrm{I}_{\mathrm{D}} @ \mathrm{~V}_{\mathrm{DS}}=8 \mathrm{~V}$ and $\mathrm{V}_{\mathrm{GS}}=10 \mathrm{~V}$ versus fluorine dose. 
(a) Fluorine ion implant

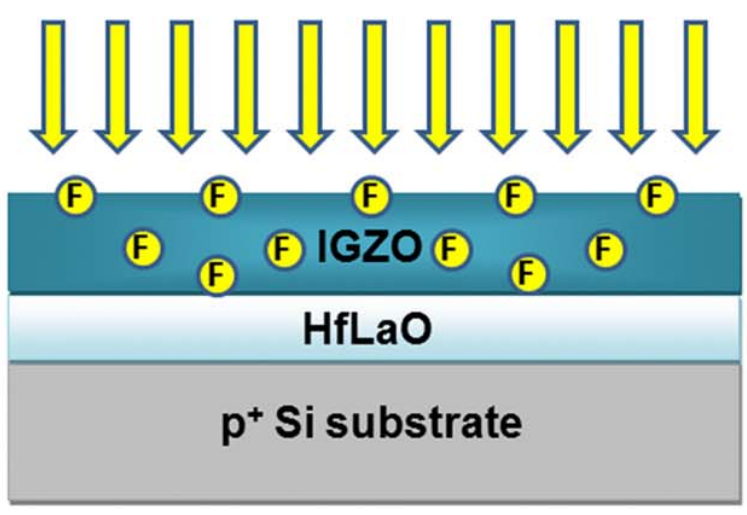

(b)

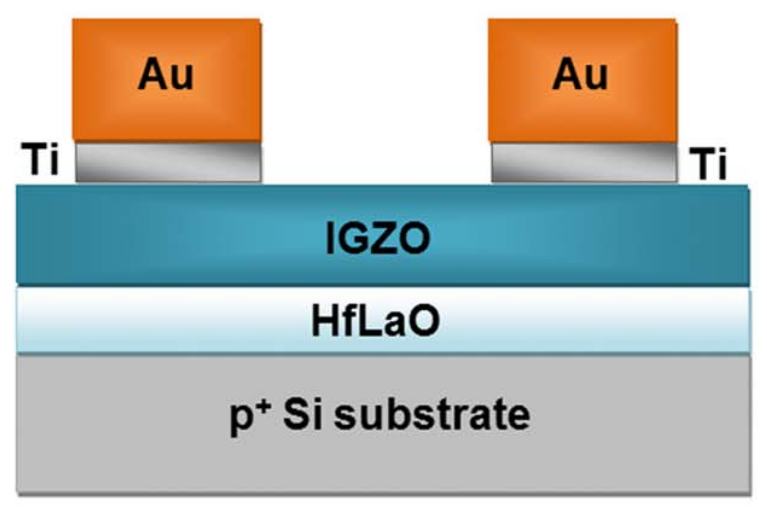

Figure 1 


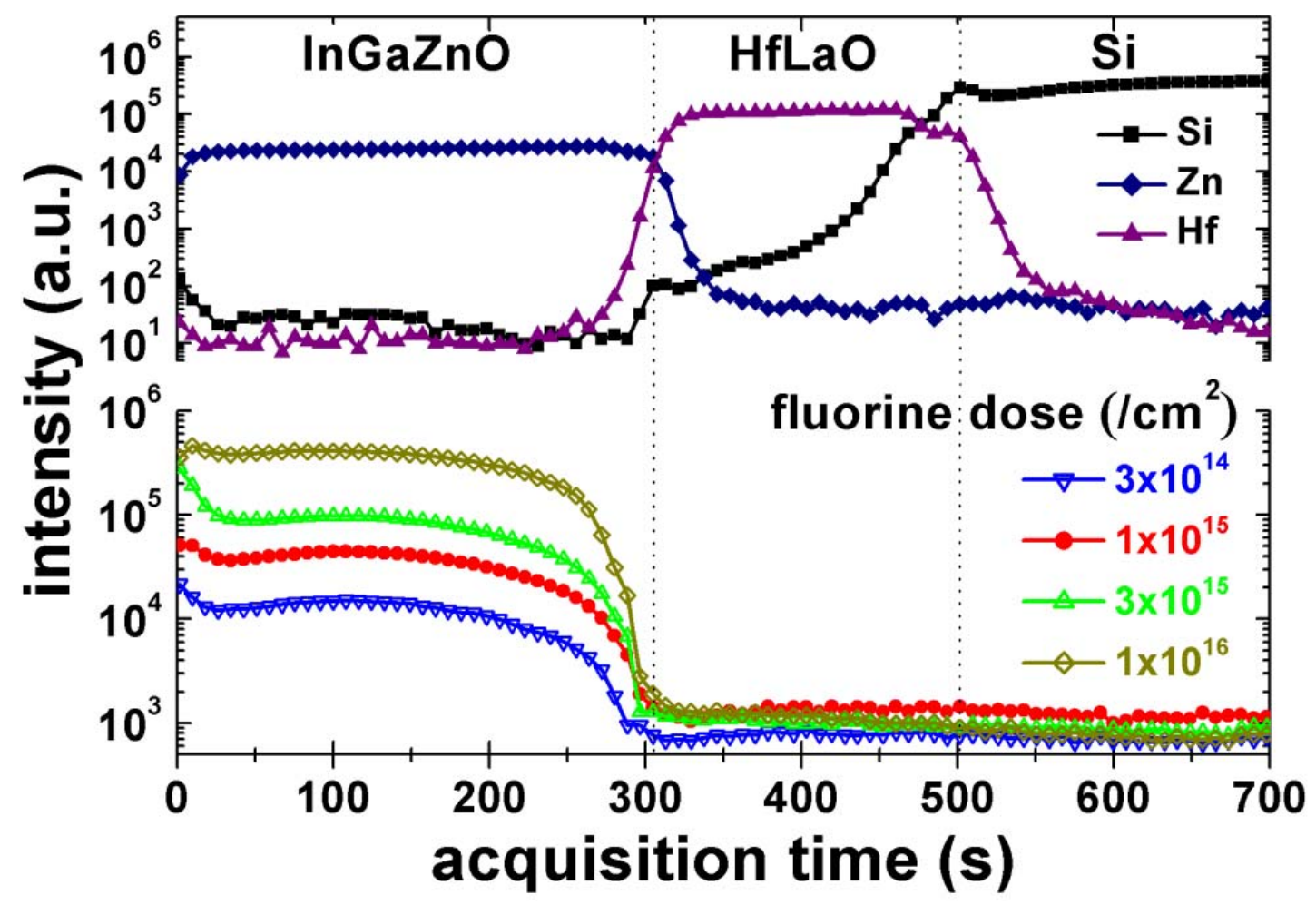

Figure 2 

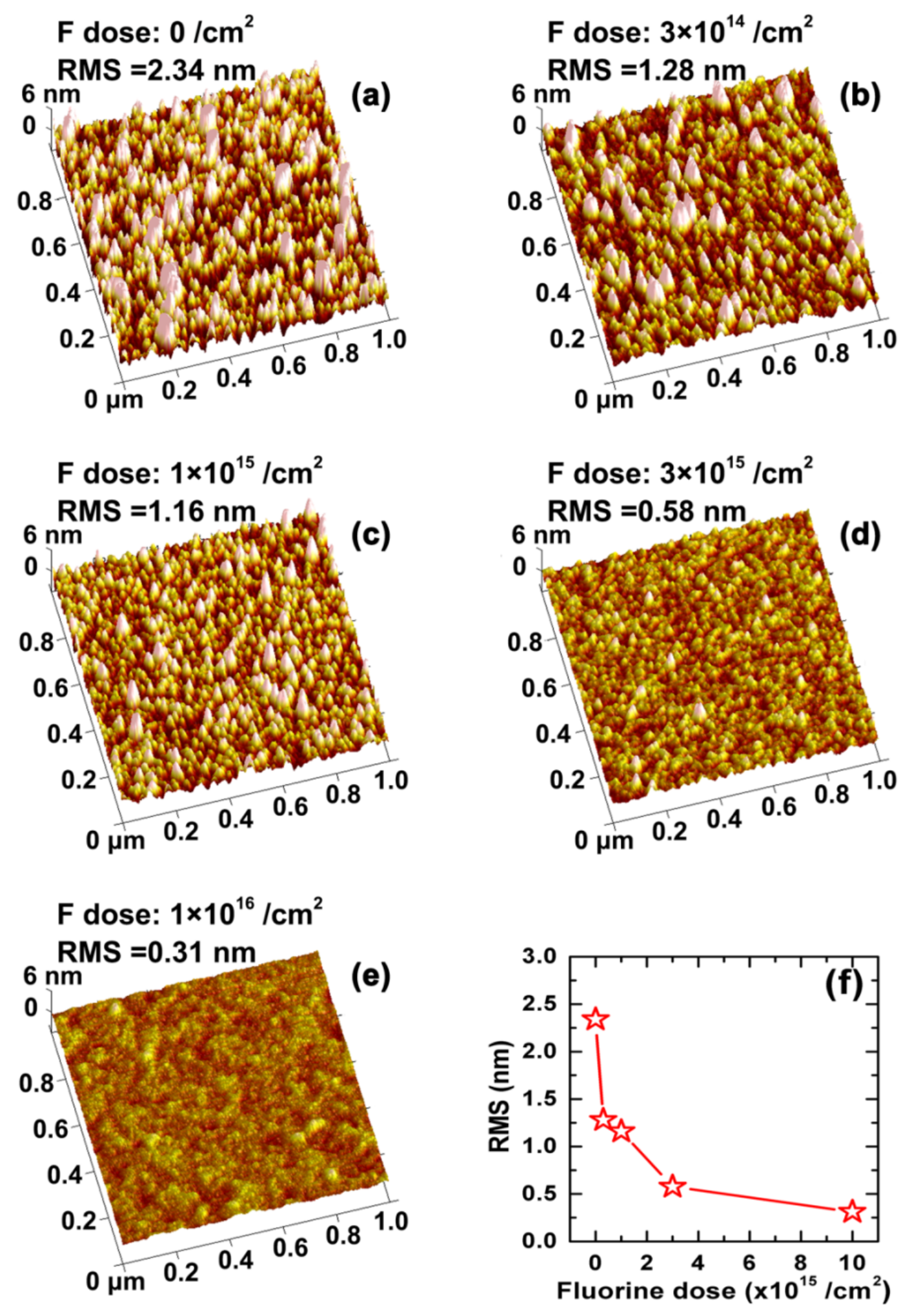

Figure 3 

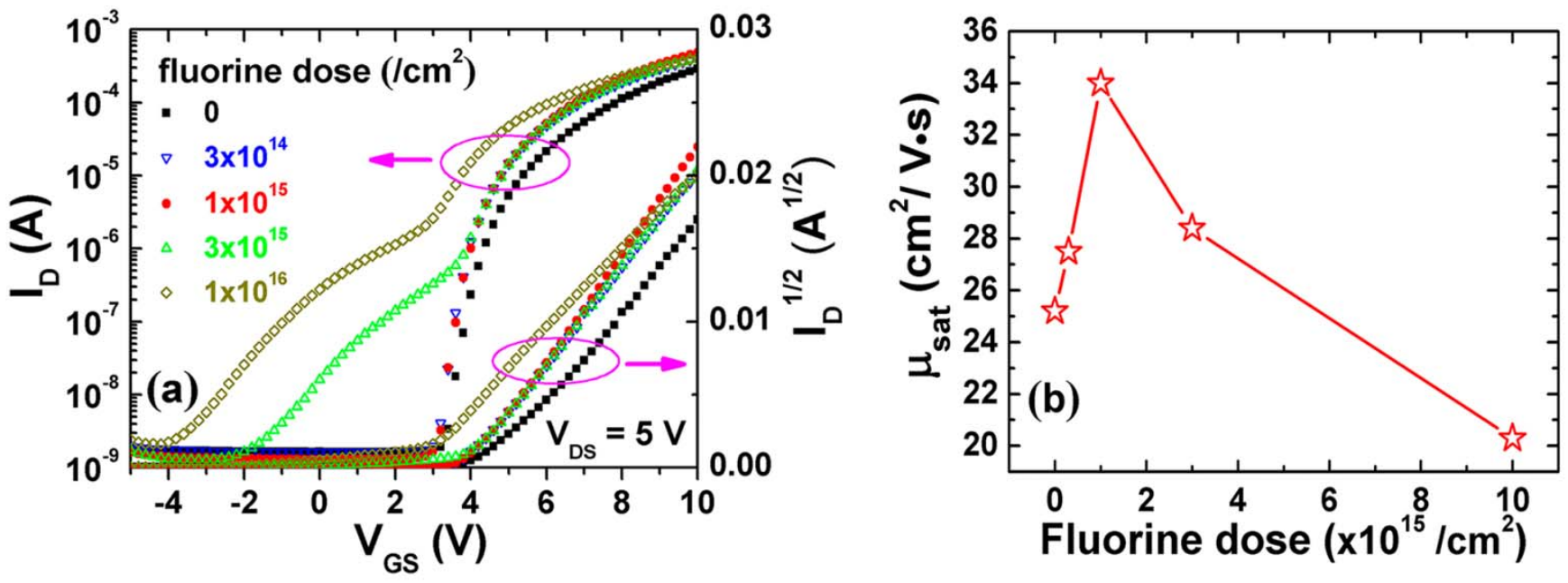

Figure 4 

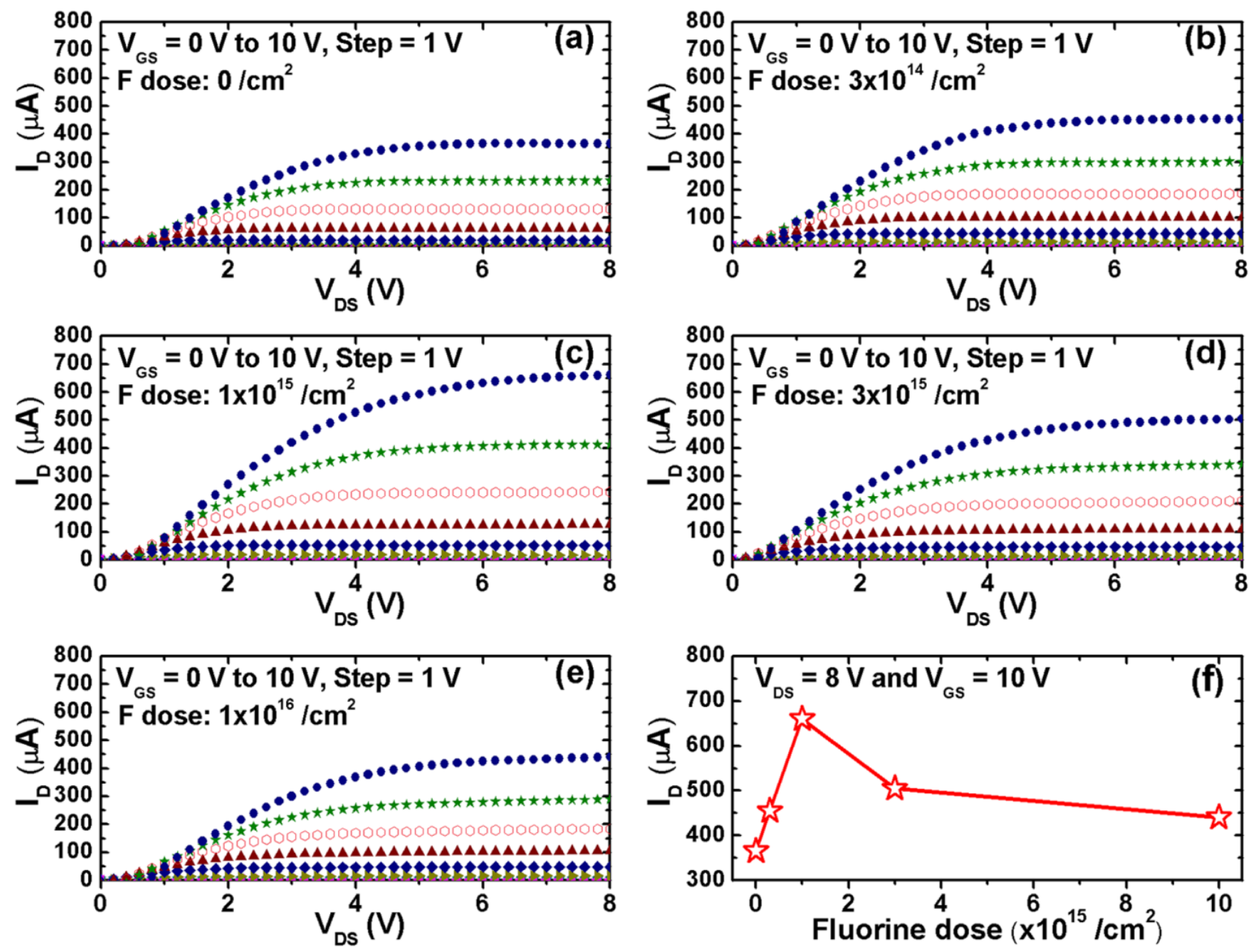

Figure 5 